\title{
Editorial: The Way Forward for JPP
}

Plasma physics is a large and fundamental area of physics, a subject that is intellectually rigorous and mathematically sophisticated and which has many applied branches. Because there are many applications, the field tends to suffer from compartmentalisation in people's minds, in terms of published output and institutional and funding arrangements. JPP will strive to be the intellectual home of those who think of plasma physics as a fundamental discipline.

While the subject is relatively old, we live in a time of exciting developments. We would like to emphasise particularly the developments in instrumentation in the laboratory, in space, and in silico, which have made measurable, observable and computable the physics that distinguishes plasma dynamics from merely fluid (or even magnetofluid) dynamics. This usually means smaller spatial and time scales and/or larger energies. Astrophysics and space physics in particular are becoming more intensively 'plasma physics' as areas of research - more so than perhaps they needed to be 10 or 20 years ago.

Similar intellectual currents flow through all of the various areas of plasma physics: multi-scale dynamics, emergence and dynamics of magnetic fields, kinetic phase-space dynamics, partition of energy between particles and fields, acceleration of particles. Many areas have seen rapid progress. A few examples:

- A number of fundamental plasma experiments have sprung up aimed at studying basic processes such as wave-wave interactions, plasma dynamos and instabilities. The broad field of "laboratory astrophysics" - which grew out of research into inertial confinement fusion, laser-plasma interactions, and high-energy-density plasmas-has made great strides in the laboratory modelling of extreme astrophysical environments such as supernovae and collisionless shocks. Vigorous numerical modelling has been a notable component of both the design and interpretation of these experiments.

- In astrophysics and space physics, the particle heating and acceleration problems (accretion discs, cosmic rays, the heliosphere) are plasma problems, which require kinetic theory, massive computing and very sophisticated observations. All of these are developing rapidly.

- Magnetic reconnection is an old fundamental problem of basic plasma physics on which there has been an impressive leap forward in understanding in the last 10 years, driven primarily by computational research and increasingly also by direct observations of heliospheric plasmas.

- Microscale plasma turbulence, which determines transport in fusion devices, has become computable (in large measure, via the development of gyrokinetic theory and codes) and measurable (via new diagnostic hardware). Simultaneously, in-situ spacecraft measurements of plasma turbulence in the solar wind and the magnetosphere, done with unprecedented spatiotemporal resolution, have made theory verifiable (and falsifiable) on an extraordinary level of detail.

In the coming years, alongside its traditional focus on basic plasma theory, JPP will welcome the kinds of contributions - including experimental, computational, astro- and space-physical - that it might previously have considered as belonging to the more "applied" branches of the subject. We hope, however, that if JPP serves as a platform for this broad range of topics, this in itself will stimulate these contributions to focus on the fundamental physical processes that are being probed.

Bill Dorland and Alex Schekochihin 